the external surface of the large $T$ antigen are required to keep it from fully dissociating from DNA, similarly to what has been observed for an archaeal hexameric helicase ${ }^{10}$

Large $\mathrm{T}$ antigen, despite having served as a model for studies of the unwinding mechanisms of hexameric helicases for years, continues to surprise scientists with its abilities. It will be interesting to see whether other hexameric helicases share these abilities, or if there are specific differences between them. In the meantime, Yardimci et al. have greatly advanced our understanding of how helicases unwind long stretches of DNA in the presence of competing cellular processes.

Michael A. Trakselis and Brian W. Graham are in the Department of Chemistry, University of Pittsburgh, Pittsburgh,
Pennsylvania 15260, USA.

e-mail:mtraksel@pitt.edu

1. Yardimci, H. et al. Nature 492, 205-209 (2012).

2. Fu, Y. V. et al. Cell 146, 931-941 (2011).

3. Tanner, N. A. et al. Nature Struct. Mol. Biol. 15 , 170-176 (2008).

4. Manosas, M., Spiering, M. M., Zhuang, Z., Benkovic, S. J. \& Croquette, V. Nature Chem. Biol. 5, 904-912 (2009).

5. Hamdan, S. M. Loparo, J. J., Takahashi, M. Richardson, C. C. \& van Oijen, A. M. Nature 457, 336-339 (2009).

6. Gai, D., Zhao, R., Li, D., Finkielstein, C. V. \& Chen, X. S. Cell 119, 47-60 (2004).

7. Gomez-Lorenzo, M. G. et al. EMBO J. 22, 6205-6213 (2003)

8. Wessel, R., Schweizer, J. \& Stahl, H. J. Virol. 66, 804-815 (1992)

9. Bochman, M. L. \& Schwacha, A. Nucleic Acids Res. 38, 6078-6088 (2010)

10.Graham, B. W., Schauer, G. D., Leuba, S. H. \& Trakselis, M. A. Nucleic Acids Res. 39, 6585-6595 (2011).
A class of fluorescent organic molecule has been designed that enables highly efficient light-emitting diodes to be made. The devices may turn out to be competitors to their conventional analogues. SEE LETTER P.234

\section{BRIAN D'ANDRADE}

$\mathrm{E}$ lectronic displays are almost ubiquitous, not least because they are the means by which people interact with the Internet. The mobile display market has seen tremendous growth ${ }^{1}$, for example, with more than 1 billion mobile phones being sold in 2006 . Liquid-crystal displays are a dominant technology for display applications, but organic light-emitting diodes (OLEDs) have steadily been incorporated into mobile devices and are increasingly being used in televisions. On page 234 of this issue, Uoyama et al. ${ }^{2}$ report a type of OLED that may offer an alternative to its conventional equivalents.

One of the unique features of an OLED display is that every pixel has an OLED, which is essentially a micrometre-sized light source. The millions of red, green and blue OLEDs in a display all have to be efficient, manufacturable with good yields and capable of providing a highly consistent light output. These requirements have been achieved by improving the organic materials used in OLEDs.

One of the key drivers of OLED technology is novel chemistry. New molecules constantly offer surprises that challenge conventional wisdom and offer alternative solutions. Tang and colleagues ${ }^{3}$ introduced the world to modern OLED designs 25 years ago. However, the devices were poor at converting input electric current to light because they incorporated inefficient fluorescent organic materials. Then, in 1998, a team led by Forrest (a physicist) and Thompson (a chemist) introduced ${ }^{4}$ devices with phosphorescent molecules that enabled almost $100 \%$ efficient conversion. Phosphorescent molecules differ from their fluorescent analogues in that they contain a heavy metal atom such as iridium.

This work led to an explosion of phosphorescent materials that enabled many desirable characteristics, such as a variety of colours, long operational lifetimes and high efficiencies. However, it remains difficult to produce a single display that uses blue, green and red phosphorescent devices, because the operational lifetimes and colour characteristics of some phosphorescent devices do not meet the strict performance requirements for display applications. It is important to solve these problems because phosphorescent materials are the most promising materials for displays, with the potential to deliver nearly $100 \%$ efficiency. Now, Uoyama et al. describe how they have made efficient OLEDs that use a special type of fluorescent material.

When an organic molecule absorbs electrical energy, it moves from its original state to a higher-energy (excited) state. For high-efficiency devices, the excited molecule preferably sheds the extra energy by producing a photon of light when it returns to its original, unexcited state. The molecular transition from excited to unexcited states has a few strict rules related to quantum-mechanical conditions that must be satisfied. These rules prevent every excited molecule from emitting a photon and returning to an unexcited state. As a result, fluorescent materials used in OLEDs often generate heat instead of light. Phosphorescent materials break those rules by using a heavy metal atom, such as iridium, bonded to the organic materials (typically comprising carbon, nitrogen and hydrogen) in an individual molecule. This makes it significantly more likely that after absorbing electrical energy, a phosphorescent molecule can efficiently transition from a high energy state to a low one by emitting a photon.

Uoyama and colleagues developed fluorescent molecules that can achieve high efficiency by using heat instead of a heavy metal atom. They convincingly demonstrated the molecules' potential by making highly efficient fluorescent OLEDs with a performance comparable to that of phosphorescent OLEDs. Their fluorescent molecules are based on carbazolyl dicyanobenzene and as such have the potential to be synthesized at low cost, Uoyama et al. believe, because the synthetic process does not require rare metal catalysts or rare heavy metal atoms. Furthermore, the OLEDs can emit multiple colours, from skyblue (with a peak wavelength at 473 nanometres) to orange (577 $\mathrm{nm})$.

There are many challenges ahead before these molecules can be fully accepted into production. The emissive colours have to be developed for display applications; the operational lifetime of devices that incorporate the molecules needs to be comparable to, or preferably better than, state-of-the-art devices (possibly the hardest challenge); and the ability to manufacture products based on the new OLEDs must be proven. The molecules are not certain to replace existing ones, but they may be shaped into contenders in terms of the cost and quality of desirable performance characteristics. Indeed, perfecting the new molecules will take time and effort, but Chihaya Adachi, who leads the work and is a co-author of the study ${ }^{2}$, has the experience needed to help his team to overcome these obstacles. .

Brian D'Andrade is at Exponent, Inc., 1150 Connecticut Avenue NW, Suite 1100, Washington DC 20036, USA.

e-mail:bdandrade@exponent.com

1. Li, Z., Bhowmik, A. K. \& Bos, P. J. in Mobile Displays: Technology and Applications (eds Bhowmik, A. K., Li, Z. \& Bos, P. J.) 2 (Wiley, 2008).

2. Uoyama, H., Goushi, K., Shizu, K., Nomura, H. \& Adachi, C. Nature 492, 234-238 (2012).

3. Tang, C. W. \& VanSlyke, S. A. Appl. Phys. Lett. $\mathbf{5 1}$ 913-915 (1987).

4. Baldo, M. A. et al. Nature 395, 151-154 (1998) 